Noise Properties of Rectifying Nanopores

M. R. Powell, N. Sa, M. Davenport, K. Healy, I.

Vlassiouk, S. E. Letant, L. A. Baker, Z. S. Siwy

March 17, 2011

Journal of Physical Chemistry C 
This document was prepared as an account of work sponsored by an agency of the United States government. Neither the United States government nor Lawrence Livermore National Security, LLC, nor any of their employees makes any warranty, expressed or implied, or assumes any legal liability or responsibility for the accuracy, completeness, or usefulness of any information, apparatus, product, or process disclosed, or represents that its use would not infringe privately owned rights. Reference herein to any specific commercial product, process, or service by trade name, trademark, manufacturer, or otherwise does not necessarily constitute or imply its endorsement, recommendation, or favoring by the United States government or Lawrence Livermore National Security, LLC. The views and opinions of authors expressed herein do not necessarily state or reflect those of the United States government or Lawrence Livermore National Security, LLC, and shall not be used for advertising or product endorsement purposes. 


\title{
Noise Properties of Rectifying Nanopores
}

M.R. Powell, N. Sa, M. Davenport, K. Healy, I. Vlassiouk, S. E. Létant, L.A.

Baker, Z.S. Siwy

Department of Physics and Astronomy, University of California, Irvine CA 92697

Department of Chemistry, Indiana University, Bloomington, IN 47405-7102

Oak Ridge National Laboratory, Oak Ridge, TN 37831, USA

Lawrence Livermore National Laboratory, Livermore, CA 94550

\begin{abstract}
Ion currents through three types of rectifying nanoporous structures are studied and compared for the first time: conically shaped polymer nanopores, glass nanopipettes, and silicon nitride nanopores. Time signals of ion currents are analyzed by power spectrum. We focus on the low-frequency range where the power spectrum magnitude scales with frequency, $f$, as $1 / f$. Glass nanopipettes and polymer nanopores exhibit non-equilibrium $1 / f$ noise, thus the normalized power spectrum depends on the voltage polarity and magnitude. In contrast, $1 / \mathrm{f}$ noise in rectifying silicon nitride nanopores is of equilibrium character. Various mechanisms underlying the voltagedependent $1 / f$ noise are explored and discussed, including intrinsic pore wall dynamics, and formation of vortices and non-linear flow patterns in the pore. Experimental data are supported by modeling of ion currents based on the coupled Poisson-Nernst-Planck and Navier Stokes equations. We conclude that the voltage-dependent $1 / f$ noise observed in polymer and glass asymmetric nanopores might result from high and asymmetric electric fields inducing secondary effects in the pore such as enhanced water dissociation.
\end{abstract}




\section{INTRODUCTION}

Single nanopores in polymer, silicon nitride ( $\mathrm{SiN}$ ) and silica films have been studied by researchers from various fields as the basis for single molecule sensors and as a model system for studying processes occurring at the nanoscale. ${ }^{1,2,3}$ Information about processes occurring inside a nanopore is typically obtained by measuring ion current, which acts as a microscopic view for interactions between passing ions or molecules and the pore walls. To study ionic transport through nanopores, average signals in the form of current-voltage curves as well as transient recordings of ion current in time are acquired. Rectifying current-voltage curves reflect the existence of a broken symmetry of the intrinsic electrochemical potential of the nanopore ${ }^{4,5}$ Ion current fluctuations and discrete pore openings and closings carry information about intrinsic dynamics of ion channels ${ }^{6}$, nanopores ${ }^{7}$, or transient formation of precipitates. ${ }^{8}$ Transient pore blockages are also formed by translocating molecules and particles whose size is similar to the pore diameter. ${ }^{1,2,3,9,10,11}$

In this article we study noise properties of ion currents recorded in single polymer and $\mathrm{SiN}$ nanopores as well as in glass nanopipettes. We focus on the low-frequency, $\mathrm{f}$, noise seen in the power spectra as $1 / f^{\alpha}$. This noise, typically called $1 / f$ noise $e^{12,13}$, appears in the frequency regime relevant for detecting single molecules and has been widely studied as a function of pore size, ionic concentration and applied voltage. ${ }^{14,15,16}$ Various nanoporous structures have been reported to exhibit different properties of $1 / f$ noise. SiN non-rectifying nanopores are characterized by equilibrium $1 / \mathrm{f}$ noise. This means the normalized noise, defined as the magnitude of the power spectrum at a chosen frequency (typically $1 \mathrm{~Hz}$ ) divided by the squared value of a current $\left(\mathrm{S}(1 \mathrm{~Hz}) /<1>^{2}\right)$, is constant and voltage-independent. ${ }^{14,15} \mathrm{~A}$ report from another group showed rectifying SiN pores which also produced equilibrium $1 / \mathrm{f}$ noise. ${ }^{17}$ These results are in contrast to the recently reported system of single conically shaped nanopores in polymer films which rectified current and exhibited non-equilibrium $1 / f$ noise: the normalized noise magnitude at $1 \mathrm{~Hz}, \mathrm{~S}(1 \mathrm{~Hz}) /<1>^{2}$, increased with voltage in an exponential manner. ${ }^{18,19,20}$ Polymer nanopores also showed a counter-intuitive result: larger currents observed at higher $\mathrm{KCl}$ concentrations were characterized by elevated values of $S(1 \mathrm{~Hz}) /<\mid>^{2}$. ${ }^{19}$ The goal of the studies presented in this paper was to 
investigate noise properties of ion currents recorded in different types of rectifying nanopores in an effort to elucidate possible mechanisms of the non-equilibrium 1/f noise observed in polymer structures. We also hoped to establish a relationship between current-voltage curves and types of noise exhibited by transient recordings.

Three types of rectifying nanoporous structures were examined: SiN nanopores drilled with a Focused Ion Beam $(F I B)^{10,21}$, conically shaped polymer nanopores prepared by the track-etching technique ${ }^{22,23}$, and glass nanopipettes fashioned from heated quartz capillaries. ${ }^{24,25}$ Polymer nanopores and nanopipettes rectify due to the asymmetric electric potential resulting from the shape and finite surface charges of the pore walls. ${ }^{4,5,24}$ Rectification in SiN nanopores results from the gold layer at the membrane surface deposited before FIB drilling, which is characterized by a higher surface charge density in $\mathrm{KCl}$ solutions ${ }^{26}$ than the SiN pore walls. ${ }^{11}$ The hypothesis tested here states that non-equilibrium $1 / \mathrm{f}$ noise can potentially result from the dynamics of a nanopore structure. Polymer structures are soft materials which can undergo an intrinsic dynamics, especially when in solution. The dynamics of the pore walls is predicted to be regulated by the charge repulsion and pressure on the walls, which occur in a charged nanopore as a function of ionic strength of the background electrolyte. ${ }^{27}$ The pressure between the pore walls prevents the "soft" walls to come in close proximity with one another. SiN nanopores and glass nanopipettes are our model system for rigid structures and their ion currents were expected to exhibit different noise behavior than currents in the polymer nanopores. We also tested whether the formation of vortices and other nonlinear fluid phenomenon might affect the noise properties of the asymmetric nanoporous systems studied.

\section{EXPERIMENTAL METHODS}

\subsection{Preparing polymer nanopores}

Single nanopores in 12 micrometer thick polyethylene terephthalate (PET) films were prepared by the track-etching technique. ${ }^{28}$ Briefly the technique entails irradiating the films with single energetic heavy ions at the linear accelerator UNILAC (GSI, Darmstadt, Germany $)^{29}$, and subsequent chemical etching of the irradiated samples. Asymmetric etching of PET in $9 \mathrm{M} \mathrm{NaOH}$ leads to the preparation of conically shaped 
nanopores. ${ }^{22}$ Pore preparation was performed in a conductivity cell: one chamber of the cell was filled with $9 \mathrm{M} \mathrm{NaOH}$ while the other chamber contained an acidic stopping solution (Figure 1 left). The process was monitored by measuring current when voltage of $1 \mathrm{~V}$ was applied across the membrane. For the pore fabrication, a Keithley 6487 picoammeter/voltage source was employed. Once the current reached a value of $\sim 100$ $\mathrm{pA}$, a sub-10 $\mathrm{nm}$ nanopore was prepared. The nanopore diameter was measured after pore preparation in $1 \mathrm{M} \mathrm{KCl}$ by relating the pore electrical resistance with its geometry. To obtain pores with diameter larger than $10 \mathrm{~nm}$, additional etching was performed with $9 \mathrm{M} \mathrm{NaOH}$ present on both sides of the membrane. Pore sizing and all subsequent transport experiments were performed in the same conductivity cell in which the pores were prepared. Nanopores used in this study had diameters between 5 and $40 \mathrm{~nm}$. Experiments were performed primarily in $\mathrm{KCl}$ solutions buffered to $\mathrm{pH} 8$ with Tris buffer.

\subsection{Pulling glass pipettes}

Quartz capillaries (0.7 mm (I.D.) and $1.0 \mathrm{~mm}$ (O.D.), Sutter Instrument, Novato, $\mathrm{CA})$ were treated with piranha solution $\left(30 \% \mathrm{H}_{2} \mathrm{O}_{2}: \mathrm{H}_{2} \mathrm{SO}_{4}=1: 3\right.$ caution: piranha reacts violently with organic material) prior to use to remove organic contaminants. Cleaned capillaries were then fashioned into nanopipettes with a pipette puller (P-2000, Sutter Instrument, Novato, CA). Electrolyte solutions were backfilled into nanopipettes using a microfill needle (World Precision Instrument, Inc.). For recording ion currents, a $\mathrm{Ag} / \mathrm{AgCl}$ electrode was inserted into the nanopipette and a second $\mathrm{Ag} / \mathrm{AgCl}$ electrode was placed in an external solution bathing the nanopipette.

\subsection{Drilling pores in $\mathrm{SiN}$ by focused ion beam}

SiN membranes with $50 \mathrm{~nm}$ thickness were purchased from SPI Supplies, West Chester, PA and coated with $20 \mathrm{~nm}$ of gold using a sputter-coater. A Strata dual beam FIB (FEI Company, Hillsboro, OR) was then used to drill single pores in each membrane by ion beam-assisted milling, and to subsequently image the pores with the electron beam. Ion beam currents of $10 \mathrm{pA}$ were typically used. Pores evaluated in this study had diameters ranging from $40 \mathrm{~nm}$ to $80 \mathrm{~nm}$. 


\subsection{Ion current recordings}

Ion current time series were recorded at a sampling frequency of $10 \mathrm{kHz}$ and filtered with a Bessel filter of $2 \mathrm{kHz}$. An Axopatch 200B amplifier and Digidata 1322A $A / D$ converter were used for data acquisition. The voltage was changed with 50 or 100 $\mathrm{mV}$ steps from $+1000 \mathrm{mV}$ to $-1000 \mathrm{mV}$. Each recording was $2 \mathrm{~min}$ long. Current-voltage curves were found by calculating arithmetic average values of the current signals for a given voltage together with the standard deviation. Figure 1 shows photographs of conductivity cells used for studying polymer (left) and SiN nanopores (middle), and the electrode assembly for investigating nanopipettes (right). In all cases ion current recordings were performed using two $\mathrm{Ag} / \mathrm{AgCl}$ electrodes. Since $\mathrm{Ag} / \mathrm{AgCl}$ electrodes are to a large degree non-polarizable and very stable, we could build a two-electrode set-up in which one electrode, placed at the tip opening of a conical nanopore, was grounded. The other electrode, placed at the base of the conical nanopore, was used to apply a given potential difference with respect to the ground electrode.

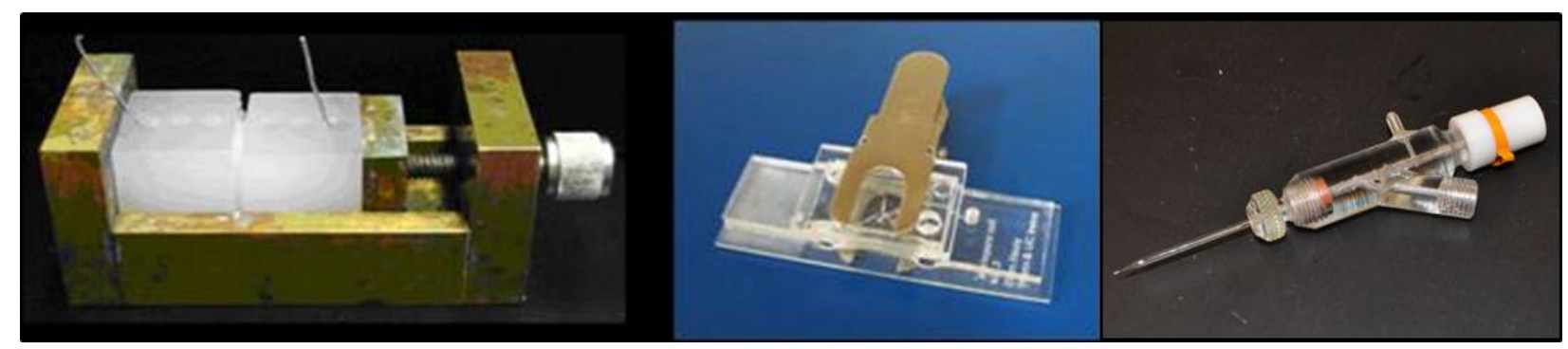

Figure 1. Photographs of conductivity cells used for measuring ion currents through single nanopores in PET (left) and single SiN nanopores (middle). Electrode assembly for measuring ion currents through nanopipettes is shown in the right panel.

\subsection{Numerical solutions of the Poisson-Nernst-Planck and Navier-Stokes equations}

Ion currents through nanopores were modeled using a continuum approach with Poisson-Nernst-Planck (PNP) equations. In some cases, the PNP approach was 
coupled with the Navier Stokes equations. The equations were solved using the COMSOL Multiphysics 3.4 package. At the charged walls, we used a triangular mesh with $0.1 \mathrm{~nm}$ spacing. The meshing at the bulk reservoirs and at the nanochannel interior was decreased to the point where no current change was observed upon further mesh re-fining. Details of the calculations can be found elsewhere. ${ }^{30,31}$

\section{RESULTS AND DISCUSSIONS}

\subsection{Polymer nanopores}

Figures 2A-4A show current-voltage curves of a $7 \mathrm{~nm}$ in diameter PET nanopore before (Figure $2 \mathrm{~A}$ ) and after its enlargement to $11 \mathrm{~nm}$ and $14 \mathrm{~nm}$ diameter (Figures $3 \mathrm{~A}$ and $4 \mathrm{~A}$, respectively), recorded at $\mathrm{pH} 8$ over a range of $\mathrm{KCl}$ concentrations between 30 $\mathrm{mM}$ and $1 \mathrm{M} \mathrm{KCl}$. Values presented are averages in time of ion current time signals recorded at each voltage for two min. Signals were very stable in time so that the standard deviation did not typically exceed $\sim 5 \%$ of the average values. Conically shaped nanopores with negative surface charges are known to be cation selective and to rectify the ion current such that the preferential direction of cation current flow is from the narrow opening toward the base of the cone..$^{4,22,23,32,33}$ In our experimental configuration with the ground electrode located at the narrow opening, negative currents are larger than the positive currents. Positive currents correspond to potassium ions being driven from the large opening to the small opening of the cone. The ion current rectification is a strong function of the electrolyte concentration and the pore diameter: the pores rectify only if the pore radius is comparable to the thickness of the electrical double-layer in a given solution. ${ }^{4,5,23,32,33}$ The dependence of the ion current rectification on salt concentration is not monotonic, however, and exhibits a maximum. For very low $\mathrm{KCl}$ concentrations, when a large portion of the pore is filled primarily with counterions, the pores rectify less. ${ }^{33}$ 

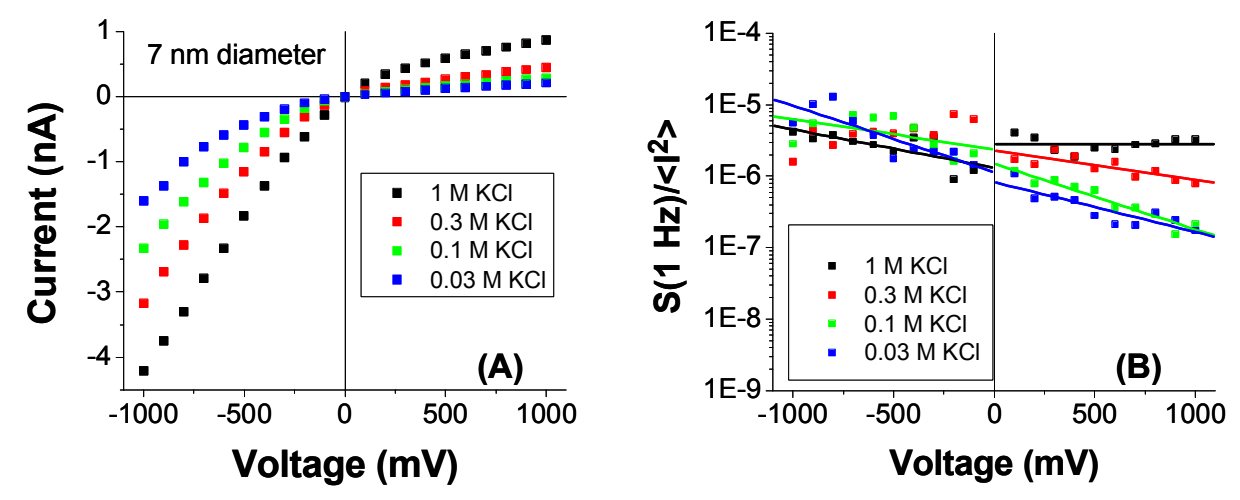

Figure 2. Current-voltage curves $(A)$ and normalized power spectra, $\left.S(1 \mathrm{~Hz}) /<l^{2}\right\rangle(B)$ for a conical PET nanopore with a tip opening of $7 \mathrm{~nm}$ in diameter for a range of $\mathrm{KCl}$ concentrations between $30 \mathrm{mM}$ and $1 \mathrm{M} \mathrm{KCl}$. The lines in (B) serve as a guide to see the trend of the $S(1 \mathrm{~Hz}) /\left\langle I^{2}>\right.$ dependence on applied voltage.
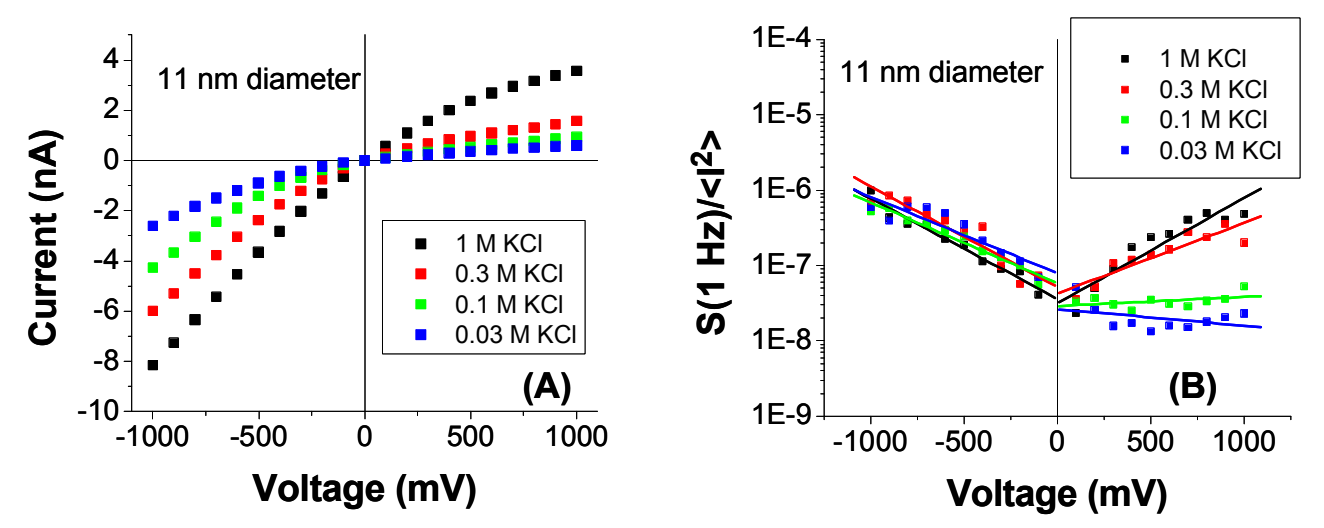

Figure 3. Current-voltage curves $(A)$ and normalized power spectra, $S(1 \mathrm{~Hz}) /\left\langle l^{2}\right\rangle$ (B) for a conical PET nanopore which was enlarged from $7 \mathrm{~nm}$ (see Fig. 2) to $11 \mathrm{~nm}$. The lines in (B) serve as a guide to see the trend of the $S(1 \mathrm{~Hz}) /\left\langle l^{2}>\right.$ dependence on applied voltage. 

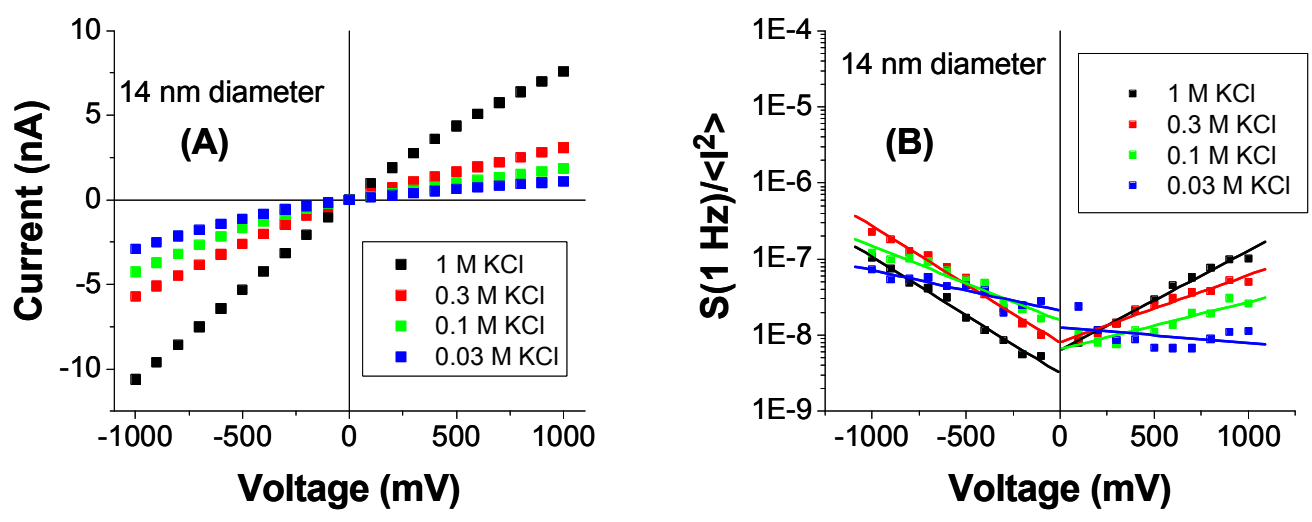

Figure 4. Current-voltage curves $(A)$ and normalized power spectra, $\left.S(1 \mathrm{~Hz}) /<l^{2}\right\rangle(B)$, for a conical PET nanopore which was enlarged from $11 \mathrm{~nm}$ (see Fig. 3) to 14 nanometers for a range of $\mathrm{KCl}$ concentrations between $30 \mathrm{mM}$ and $1 \mathrm{M} \mathrm{KCl}$. The lines in (B) serve as a guide to see the trend of the $S(1 \mathrm{~Hz}) /<l^{2}>$ dependence on applied voltage.

Noise properties of conically shaped and rectifying nanopores have recently been studied by power spectra which revealed an existence of $1 / \mathrm{f}$ noise in the lowfrequency range of the power spectrum. ${ }^{18}$ In contrast to previously reported nanopores in $\mathrm{SiN}{ }^{14,15,17}$ as well as biological pores ${ }^{13}$, rectifying conically shaped nanopores produced $1 / f$ noise of a non-equilibrium type. Figures 2B-4B show normalized power spectra presented as a magnitude of a given power spectrum at $1 \mathrm{~Hz}$ divided by the square value of the current, as a function of applied voltage. If the $1 / \mathrm{f}$ noise had equilibrium character, $S(1 \mathrm{~Hz}) /<1>^{2}$ would be voltage-independent and would fulfill the so-called Hooge formula ${ }^{34}$ (Eqn 1), which relates the magnitude of the power spectra with the number of charge carriers $N_{c}$, where $\alpha_{H}$ is the Hooge parameter determined for a given material.

$$
S(f)=\frac{I^{2} \alpha_{H}}{f N_{c}} \quad \text { Equation } 1
$$

The Hooge formula also predicts that the magnitude of the normalized noise should decrease with an increase in the number of charge carriers in the pore. In ionic solutions, the number of charge carriers is controlled by the concentration of the background electrolyte. According to the Hooge formula, currents at higher $\mathrm{KCl}$ concentrations are expected to exhibit a lower normalized noise compared to the 
currents recorded at lower $\mathrm{KCl}$ concentrations. In our system, especially for positive voltages, the dependence of $\mathrm{S}(\mathrm{f})$ on $\mathrm{KCl}$ concentration is exactly opposite to the Hooge formula predictions. At positive voltages, $S(1 \mathrm{~Hz}) /<1>^{2}$ for currents recorded in $1 \mathrm{M} \mathrm{KCl}$ is higher than values of $S(1 \mathrm{~Hz}) /<1>^{2}$ for currents in $0.1 \mathrm{M} \mathrm{KCl}$ (Figures $2 \mathrm{~B}-4 \mathrm{~B}$ ). Ionic concentrations in a conically shaped nanopore are also strongly voltage-dependent and increase with the increase of negative voltages. For positive voltages, the opposite effect is observed: an increase of the voltage magnitude leads to the formation of a depletion zone in the pore. ${ }^{18}$ Taking this effect into account, it becomes even more evident that the Hooge formula cannot describe noise properties of conically shaped nanopores.

A model which could explain the experimental observations on voltagedependent noise properties of ion currents in conical nanopores considers the existence of internal dynamics or "breathing" of the pore walls. Let us first consider two charged walls separated by a distance $d$. If $d$ is small and comparable to the thickness of the electrical double-layer, the electrostatic repulsion between the walls results in a pressure on the walls. ${ }^{27}$ If the distance becomes larger, or if the walls are in contact with a solution of higher ionic strength, the surface charges are screened, preventing the walls from "seeing" each other; as a consequence, the pressure between the walls drops to zero. We think that since the pores are made out of a soft material, they will undergo an intrinsic dynamics which will be especially pronounced during the absence of the pressure between the walls. This wall' "breathing" would occur at low frequencies and be responsible for the $1 / \mathrm{f}$ noise in ion currents observed through polymer nanopores. The noise properties change with applied voltage, because ionic concentrations in the pore are strongly-voltage dependent, as previously mentioned.

Figure 5 presents ionic concentrations inside a conically shaped nanopore for different voltages and bulk ionic concentrations, as obtained by numerically solving the Poisson-Nernst-Planck equations. ${ }^{18,30,31,35}$ The calculations were performed for a conically shaped nanopore with two openings of $7 \mathrm{~nm}$ and $500 \mathrm{~nm}$ in diameter, and a surface charge density of 0.5 elementary charge per $\mathrm{nm}^{2}$. The concentrations presented are average values in a given position along the pore axis, $x$. Potassium and chloride 
concentrations in the pore are indeed a strong function of the applied voltage, and bulk $\mathrm{KCl}$ concentration. The ionic concentrations in the pore for negative voltages are at least several times higher than the concentrations in the bulk for all bulk concentrations studied. On one hand, for positive voltages the ionic concentrations are lower than the bulk; increasing positive voltage creates a region several micrometers wide with depleted potassium ions, while chloride ions are excluded from the narrow opening almost entirely for $30 \mathrm{mM}$ and $100 \mathrm{mM}$ bulk KCl . The smaller number of ions in the nanopore provides less screening to the surface charges of the pore walls, which leads to a higher pressure difference and larger rigidity of the structure; as a consequence, positive ion currents are characterized by a lower normalized power spectrum. On the other hand, for negative voltages, higher ionic concentrations screen the charges very effectively, resulting in a "softer" and more dynamic nanopore structure.

Even for $1 \mathrm{M} \mathrm{KCl}$ background electrolyte, applying an external voltage modulates ionic concentrations in the pore. The depletion of ionic concentrations at positive voltages is much less complete, however, as compared to the situation at $30 \mathrm{mM}$ and $100 \mathrm{mM}$. Consequently, the values of $\left.S(1 \mathrm{~Hz}) /<\left.\right|^{2}\right\rangle$ at positive and negative voltages are comparable (Figure 2B).

The analysis performed with polymer PET nanopores suggests that the mechanism for non-equilibrium fluctuations could indeed be related with the intrinsic dynamics of the polymer walls, which is most pronounced when the surface charges are screened by ions in the pore. Increase of $S(1 \mathrm{~Hz}) /\left\langle\left.\right|^{2}\right\rangle$ for negative voltages is in agreement with the voltage-dependent increase of potassium and chloride concentrations for this voltage polarity.

The hypothesis on the importance of the screening of the pore wall surface charge on the properties of $1 / f$ noise is also confirmed by the dependence of $\mathrm{S}(1 \mathrm{~Hz}) /<1>^{2}$ on bulk $\mathrm{KCl}$ concentration. The dependence of $\mathrm{S}(1 \mathrm{~Hz}) /<1>^{2}$ on voltage polarity is especially pronounced for conditions at which the pore diameter is comparable to the thickness of the electrical double-layer. 

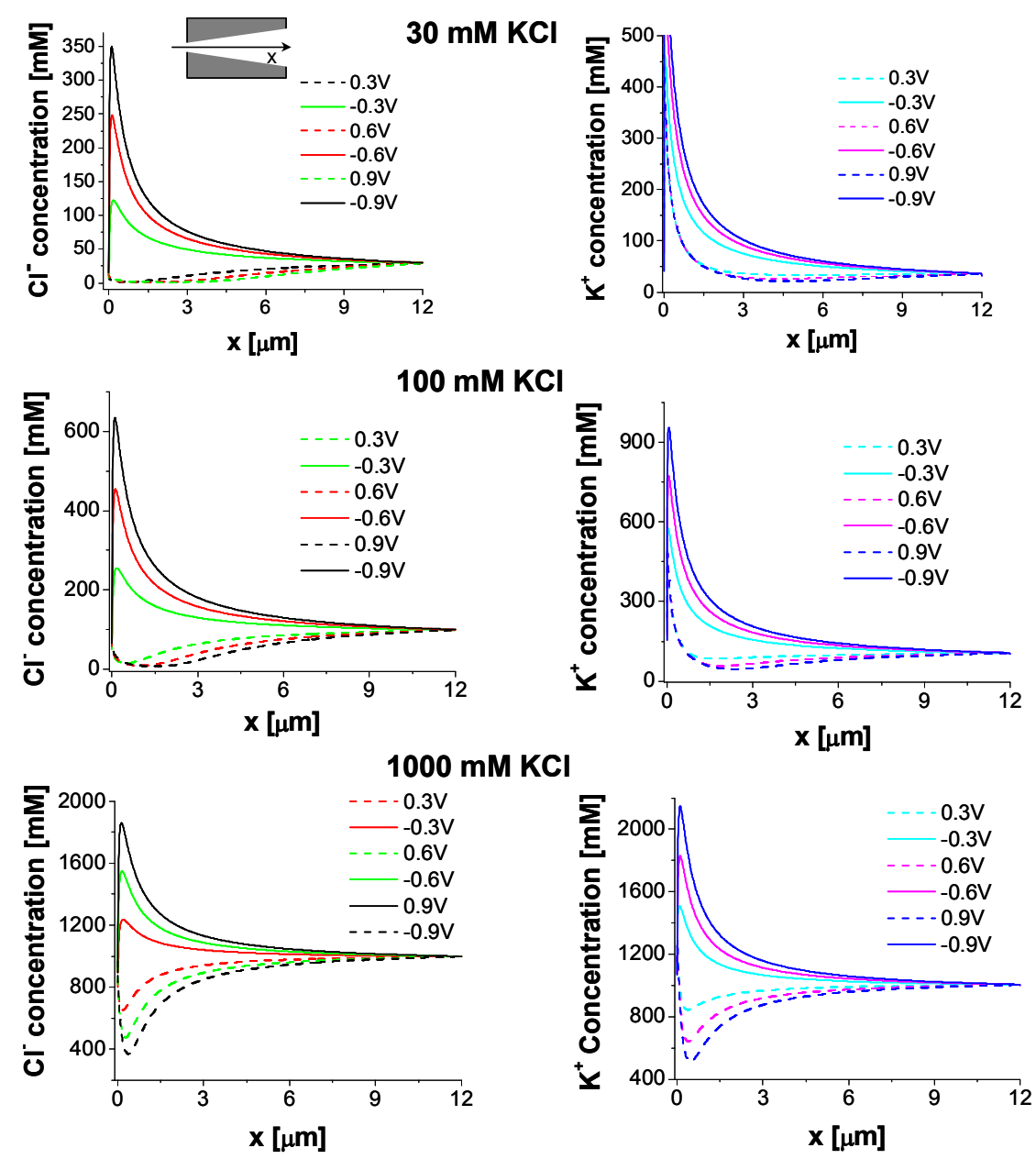

Figure 5. lonic concentrations along the pore axis $x$ in a single conically shaped nanopore with openings of 7 and $500 \mathrm{~nm}$ in diameter. Concentrations were calculated based on the Poisson-Nernst-Planck equations as described in Refs. [30,31,35]. Voltages and bulk $\mathrm{KCl}$ concentrations are indicated in the figure.

To strengthen the argument that properties of $1 / f$ noise in ion currents through conically shaped polymer nanopores are dependent on voltage polarity, we compared the magnitude of the power spectrum at $1 \mathrm{~Hz}$ for currents of the same absolute value, but recorded for voltages of the opposite polarity. Figure 6 presents the relation of $\mathrm{S}(1 \mathrm{~Hz})$ on the current value for a conically shaped nanopore with a narrow opening of $11 \mathrm{~nm}$ in diameter (the same pore studied in Figure 3). The graph indicates that currents of the same absolute value can indeed have significantly different power spectra magnitudes. 

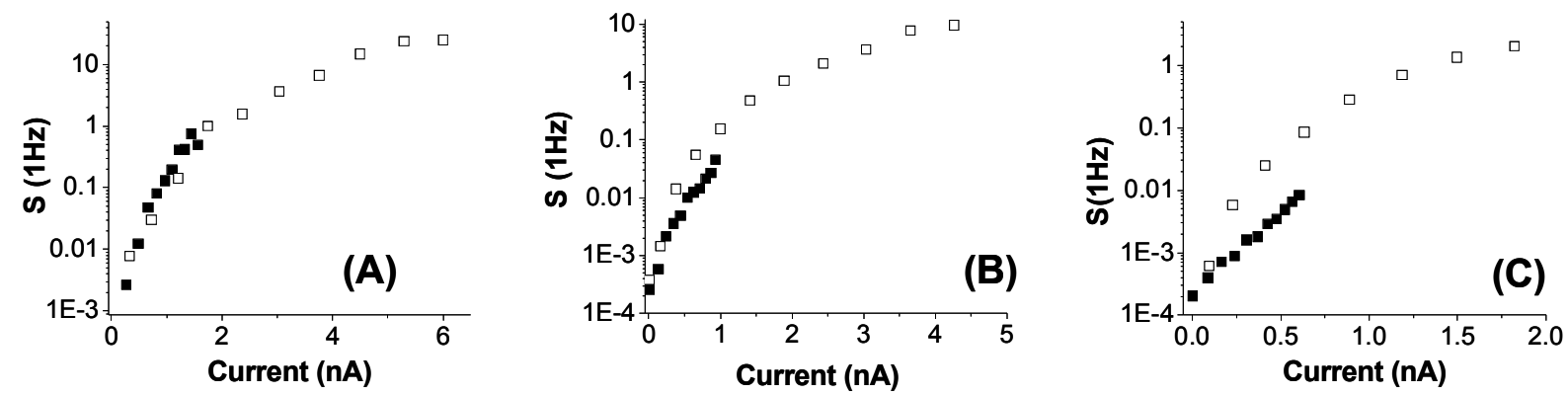

Figure 6. Magnitude of power spectrum at $1 \mathrm{~Hz}$ for ion currents recorded in: (A) $0.3 \mathrm{M}$ $\mathrm{KCl}$; (B) $0.1 \mathrm{M} \mathrm{KCl}$, and (C) $0.03 \mathrm{M} \mathrm{KCl}$, plotted as a function of ion current values. Filled squares (-) present $S(1 \mathrm{~Hz})$ for currents recorded for positive voltages; empty squares (口) are for negative currents. Positive currents are lower in magnitude so the relation of $S(1 \mathrm{~Hz})$ is presented for a shorter range of current values compared to negative currents.

To further investigate the role played by pore walls dynamics in the $1 / f$ noise properties, we studied ion currents in other rectifying systems, namely glass nanopipettes and rectifying $\mathrm{SiN}$ nanopores. Since both $\mathrm{SiN}$ and glass structures are rigid, they constitute good control systems for the testing of our hypothesis.

\subsection{Quartz nanopipettes}

Figure 7A presents current-voltage curves of a glass nanopipette with an opening of $\sim 20 \mathrm{~nm}$ in diameter recorded in $0.01 \mathrm{M}$ and $0.1 \mathrm{M} \mathrm{KCl}$ as the background electrolyte. lon current recordings were performed in the same manner as those for polymer nanopores, so that the current-voltage curves present average values over 2-min recordings. Figure 7B shows normalized power spectra of the ion current time series. We were surprised to see the same type of asymmetry of $S(1 \mathrm{~Hz}) /<1>^{2}$ with voltage polarity, as previously observed for polymer nanopores. Similar to the conical polymer pores, ion currents in the pipettes were characterized by a decreased normalized power spectrum at lower $\mathrm{KCl}$ concentrations, which is in contradiction to the Hooge formula. $\mathrm{S}(1 \mathrm{~Hz}) /<\mid>^{2}$ for negative voltages increased with an increase of the voltage magnitude, suggesting a non-equilibrium character of the $1 / \mathrm{f}$ noise. We studied three glass 
nanopipettes; all pipettes rectified the ion current at $0.1 \mathrm{M}$ and $0.01 \mathrm{M} \mathrm{KCl}$ and exhibited voltage-dependent $\mathrm{S}(1 \mathrm{~Hz})|<|>^{2}$.

It is important to mention that the degree of rectification for pipettes exceeded a factor of 5 in the voltage range between $-1 \mathrm{~V}$ and $+1 \mathrm{~V}$. It is in fact a higher rectification degree than that obtained with single conically shaped nanopores. We think the high current-voltage curve asymmetry is related to the shape of the nanopipettes. As studied by Ramirez et al. [36], the shape of nanopores strongly influences rectification properties of the structures. Nanopores with an elongated tip region, similar to the shape of the pipettes, were predicted to have superior rectification properties in comparison to tapered-cone shaped nanopores.
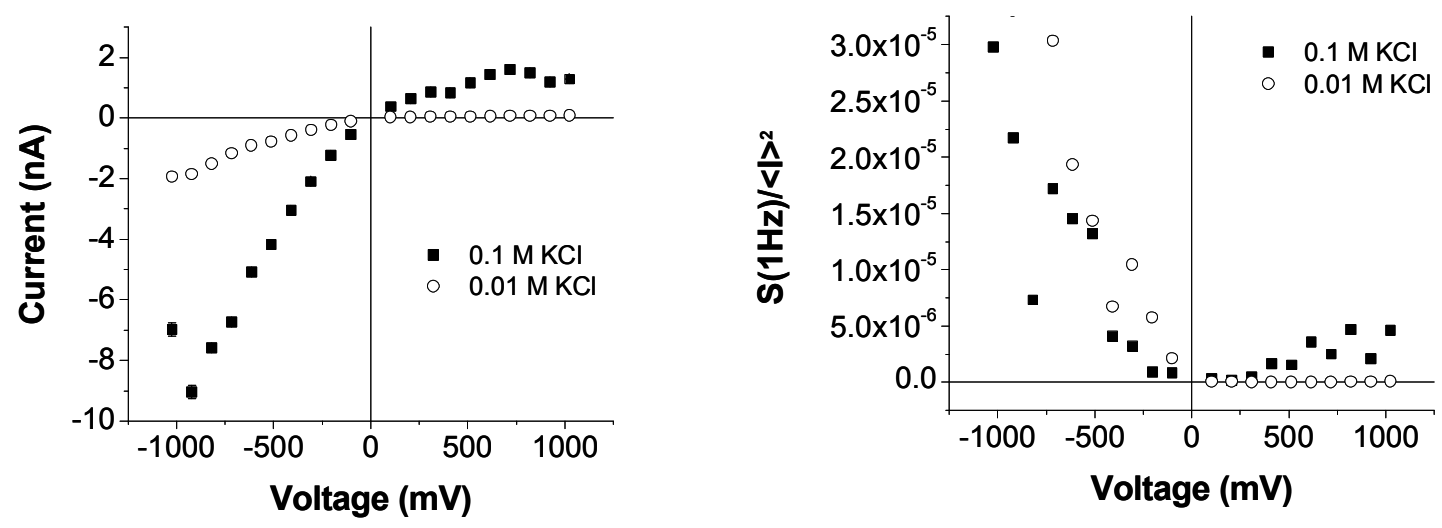

Figure 7. (A) Current-voltage curves through a single glass nanopipette with an opening diameter of $20 \mathrm{~nm}$ at $0.1 \mathrm{M} \mathrm{KCl}$ and $0.01 \mathrm{M} \mathrm{KCl}, \mathrm{pH}$ 8, together with (B) the normalized power spectra of the ion current time series.

We think that the voltage-dependent character of the normalized power spectrum of currents in glass nanopipettes cannot originate from the dynamics of the pore walls, since these structures are rigid. It is possible that the asymmetry of the normalized noise in glass pipettes and polymer nanopores might have different physical basis.

As a next step, we studied the noise properties of ion currents through rectifying SiN nanopores. 


\subsection{SiN nanopores}

In the search for physical phenomena responsible for the non-equilibrium character of the $1 / \mathrm{f}$ noise of ion currents in polymer and glass rectifying structures, we analyzed ion current time series recorded through single SiN pores prepared by FIB milling. ${ }^{21}$ Structures studied had a low aspect ratio $(\sim 1)$, thus the diameter and the length of the pores were comparable. The shape of the pores was approximated as a cylinder. In the process of the pore fabrication, however, a gold layer was deposited on one side of the membrane. A gold surface in contact with $\mathrm{KCl}$ is known to be negatively charged due to the formation of a monolayer of $\mathrm{Cl}^{-}$. The resulting surface charge density of the gold layer has been measured to be quite high, typically $0.32 \mathrm{C} / \mathrm{m}^{2} .{ }^{26}$ In contrast, the surface charge density of the SiN surface is only $\sim 30 \mathrm{mC} / \mathrm{m}^{2} .{ }^{11}$ The SiN nanopores, although geometrically symmetric, exhibit asymmetry in their electrochemical potential resulting from the difference in the surface charge density of the membrane surface and the pore walls, and consequently were predicted to rectify the ion current. Figures $8 \mathrm{~A}, \mathrm{~B}$ show numerical calculations of $\mathrm{K}^{+}$and $\mathrm{Cl}^{-}$concentrations, respectively, in a single cylindrical nanopore with one entrance highly charged. The numerically predicted current-voltage curve is presented in Figure $8 \mathrm{C}$.
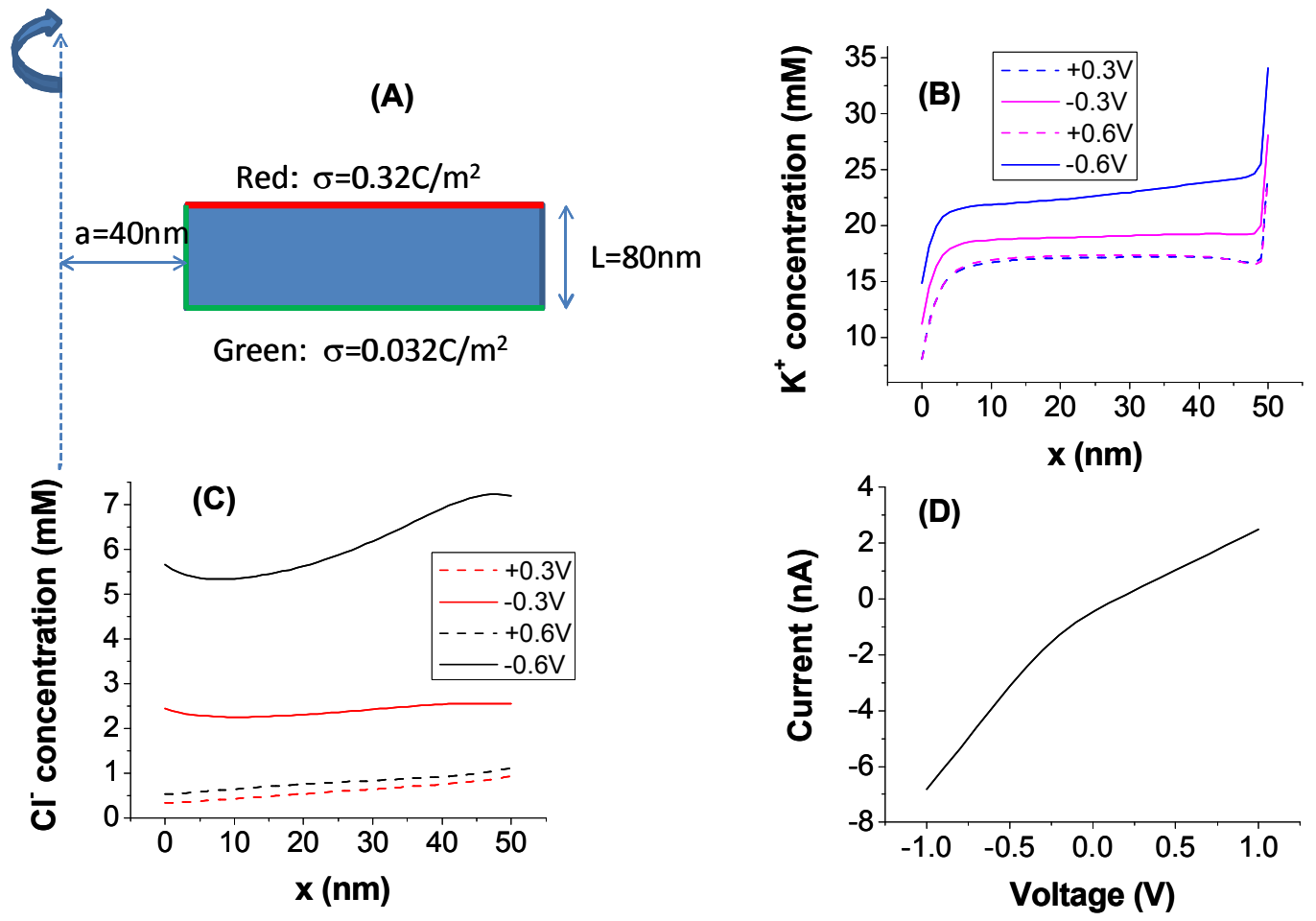
Figure 8. Ionic concentrations and current-voltage curve of a single SiN nanopore in $0.1 \mathrm{mM} \mathrm{KCl}$, modeled by the Poisson-Nernst-Planck equations. (A) Scheme of surface charges and geometry of the SiN nanopore that was modeled, (B) and (C) present profiles of potassium and chloride concentrations along the pore axis, respectively. (D) Calculated current-voltage curve. The modeled nanopore had radius, a, of $40 \mathrm{~nm}$ and length, $L$, of $80 \mathrm{~nm}$.

Figure 9 presents normalized power spectra, $\mathrm{S}(1 \mathrm{~Hz})|<|>^{2}$, of ion currents through a single SiN pore recorded in $0.3 \mathrm{mM} \mathrm{KCl}$, together with the current-voltage curve of the examined nanopore. Studied SiN nanopores were much shorter compared to the polymer and glass nanopipettes thus the measured currents are significantly higher. The range of power spectrum with $1 / f$ noise of ion current time signals is voltage dependent and extends over two orders of magnitude for $+/-500 \mathrm{mV}$. The normalized power spectra however did not show any significant voltage-dependence, which is in contrast with the results described above for PET pores and glass nanopipettes. For some SiN nanopores we observed higher levels of the normalized noise for positive currents which in rectifying nanopores were smaller than currents recorded for negative voltages. This property is in agreement with previously reported rectifying SiN nanopores whose noise characteristics could be described by the Hooge formula. ${ }^{17}$
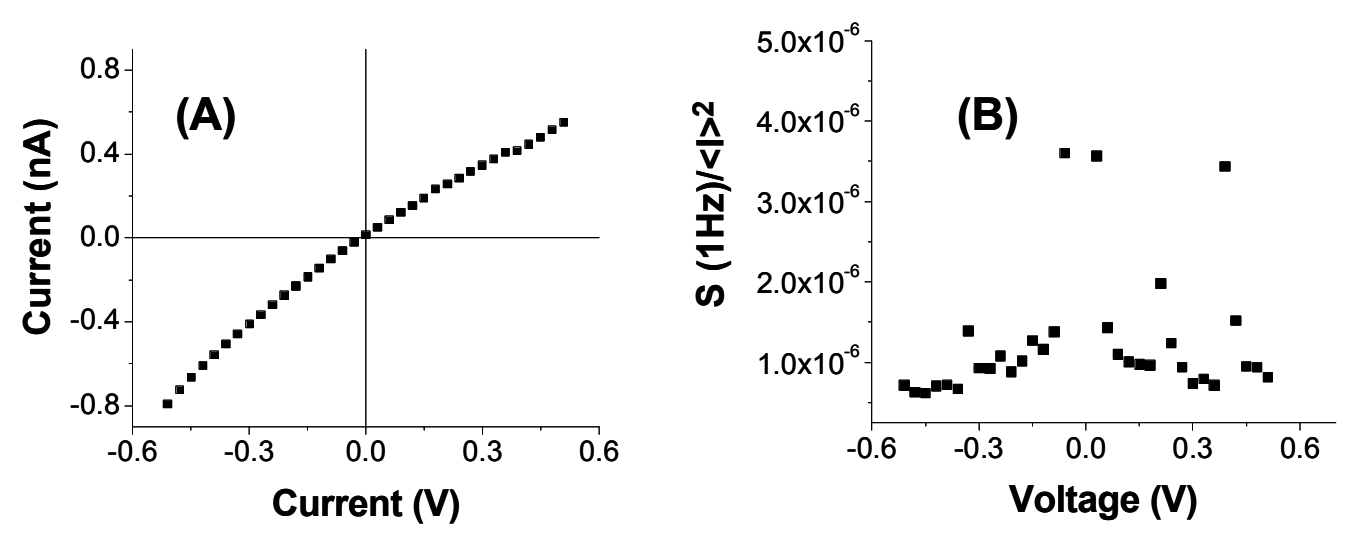

Figure 9. Properties of ion current through a single $80 \mathrm{~nm}$ diameter FIB-drilled pore in a $50 \mathrm{~nm}$ thick SiN film, recorded in $0.3 \mathrm{mM} \mathrm{KCl}$. (A) Current-voltage curves, and (B) normalized power spectra $S(1 \mathrm{~Hz}) /<\left.\right|^{2}>$. 


\subsection{Noise properties of polymer nanopores as a function of solution temperature and viscosity}

The dependence of ion current noise properties on voltage polarity observed for glass nanopipettes indicated that the dynamics of pore walls in polymer pores might not be the only phenomenon contributing to the $1 / f$ noise properties. Another possibility for the enhanced levels of noise observed in nanoporous structures was proposed by Rubinstein ${ }^{37}$, and recently demonstrated for planar nanofluidic systems. ${ }^{38}$ With high electric fields applied across the membrane, there is a possibility of formation of fluid vortices at the pore opening, which was found to be dependent on the magnitude and polarity of the applied voltage.

Some rectifying bipolar diode systems were also shown to have a peculiar distribution of fluid flow along the pore radius so that sufficiently far away from the pore walls, the fluid could change the direction of its movement. ${ }^{39}$ This change of direction might potentially induce instabilities of ion current flow. The fluid flow i.e. osmosis occurs because ions can drag water molecules when moving down their electrochemical gradient. At a negatively charged nanopore filled predominantly with cations, one therefore expects the fluid flow to occur towards the negatively biased electrode. In our modeling, performed by numerically solving the coupled PoissonNernst-Planck (PNP) and Navier-Stokes (NS) equations, we looked at the values of the fluid flow velocity in a single conically shaped nanopore. Due to significantly more demanding computational resources required for solving the coupled PNP and NS equations (vs only PNP), we model our system with shorter pores, i.e. $1 \mu \mathrm{m}$ in length with $6 \mathrm{~nm}$ and $100 \mathrm{~nm}$ opening diameters. Negative values of the velocity indicate the expected direction of osmosis. Figure 10 presents the radial distribution of velocity vectors at different positions of the pore axis, and velocities at one pore cross-section with radius of $50 \mathrm{~nm}$, located $0.5 \mu \mathrm{m}$ away from the narrow opening of the pore. Similar

to the case of a bipolar diode ${ }^{39}$, at low $\mathrm{KCl}$ concentrations, the fluid flow changes its direction $\sim 10 \mathrm{~nm}$ from the pore walls so that the calculated velocity becomes positive. At $1 \mathrm{M} \mathrm{KCl}$, the inversion of the fluid flow direction was not observed (Figure 10). It should be noted that the flow inversion does not happen along the whole pore length: at the 
small radii region at the pore tip, the solution still flows following the expected electroosmotic direction (towards the negatively biased electrode).

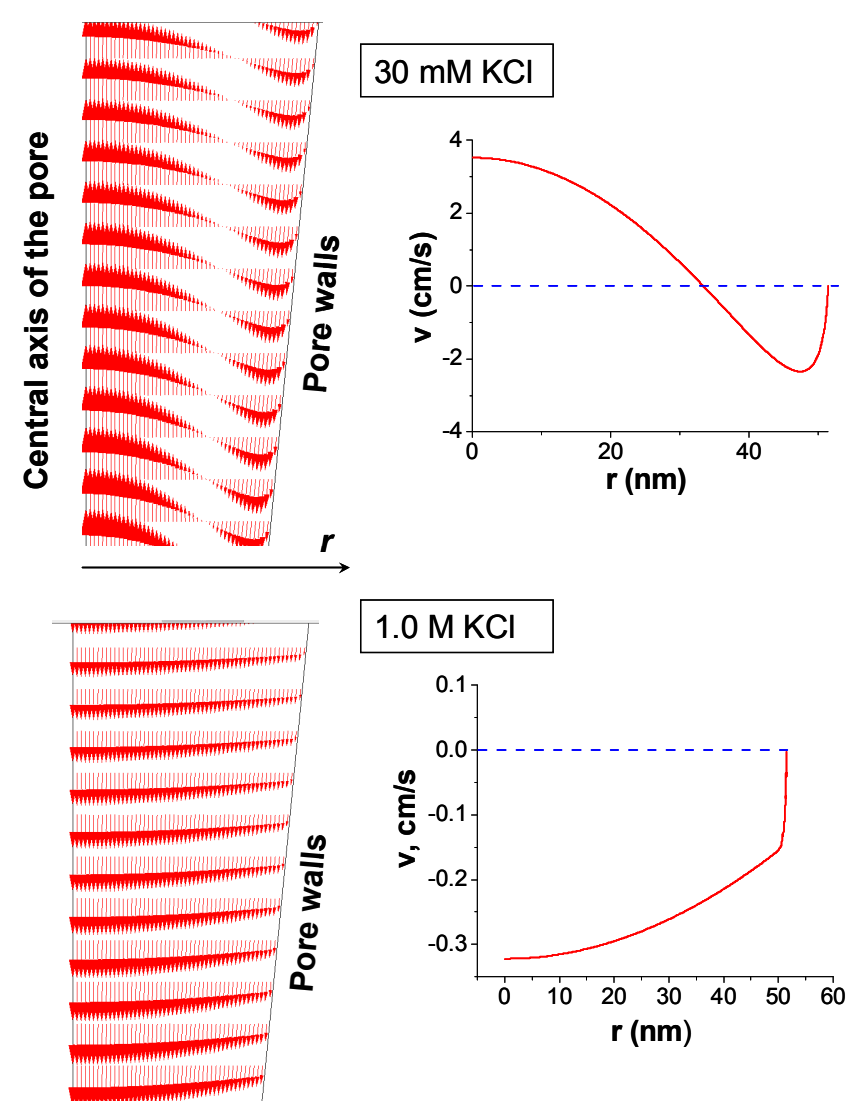

Figure 10. Calculations of the velocity vectors in a single conically shaped nanopore, performed by numerically solving the coupled Poisson-Nernst-Planck and Navier Stokes equations. At $30 \mathrm{mM} \mathrm{KCl}$, the fluid flow changes its direction $\sim 10 \mathrm{~nm}$ away from the pore walls thus the fluid moves towards the positively biased electrode. Position $r=0$ corresponds to the middle of the pore. The modeled nanopore was $1 \mu \mathrm{m}$ long, and had opening diameters of 6 and $100 \mathrm{~nm}$.

If vortices and fluid dynamics were indeed responsible for the voltage dependent magnitude of the $1 / f$ noise, an increase of the solution viscosity and lowering of the temperature would decrease $\left.\mathrm{S}(1 \mathrm{~Hz}) /\left.\right|^{2}\right\rangle$. The solution viscosity was changed by adding glycerol in the amount of $40 \%$ by weight, which increased the solution viscosity four times in comparison to an aqueous $\mathrm{KCl}$ solution. ${ }^{40}$ We analyzed three experimental situations (Figure 11): (i) the glycerol solution was present on one side of the membrane 
with the small opening; the other side of the membrane was in contact with a water solution of $\mathrm{KCl}$, (ii) the glycerol solution was present on the side of the membrane with the large opening, and (iii) symmetric conditions with glycerol solutions on both sides of the membrane. As expected, addition of glycerol lowered the ion current, which is especially pronounced in the symmetric electrolyte case (iii). In the cases (ii) and (iii) the pore rectified the current and the normalized power spectrum of ion currents was still higher for negative voltages, quantitatively similar to the case with an aqueous $\mathrm{KCl}$ solution. Thus glycerol did not influence the values of $S(1 \mathrm{~Hz}) /<1>^{2}$.
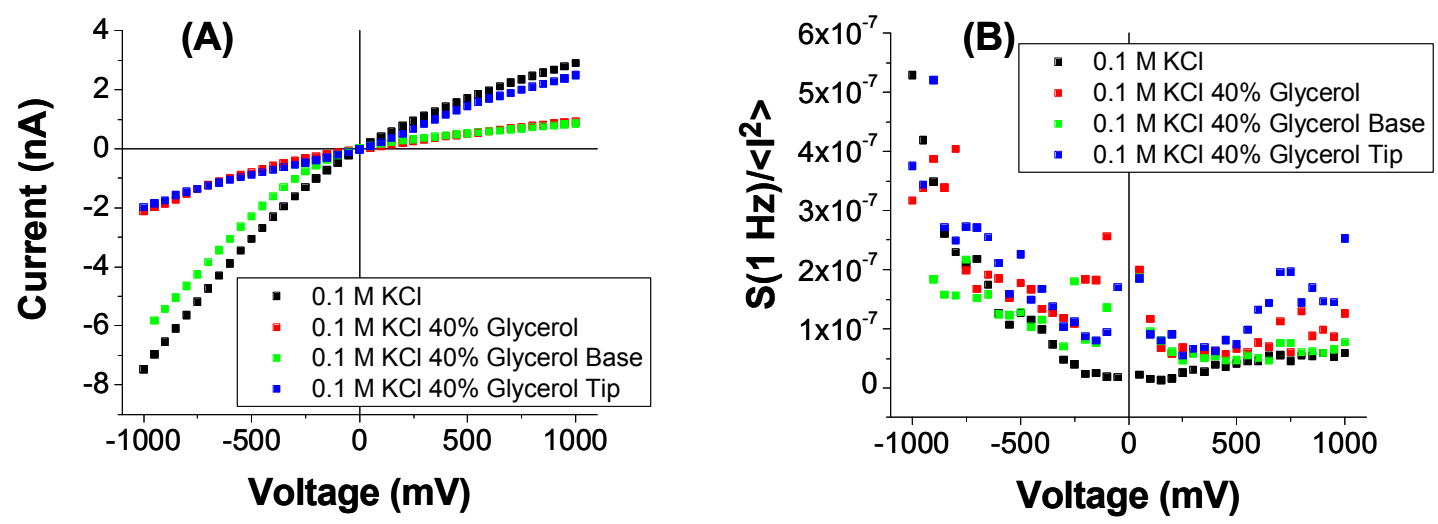

Figure 11. Effect of glycerol on ion current through a single conically shaped nanopore with a small opening of $38 \mathrm{~nm}$ in diameter. (A) Current-voltage curves measured with the glycerol $\mathrm{KCl}$ solution placed on the large or/and small opening of the pore, as indicated in the legend. (B) Normalized power spectrum at $1 \mathrm{~Hz}$ for ion current signals whose averages are shown in $(A)$.

Our measurements indicated that adding glycerol to the side of the membrane with the large opening enhanced the rectification properties of conically shaped nanopores. With glycerol at the base and for positive voltages, potassium ions are sourced from the higher viscosity solution, causing the currents to decrease even more, compared to the $\mathrm{KCl}$ water solution. The small opening of the pore is still in contact with a low density solution, and consequently, the negative currents remain almost unaffected by the glycerol in this electrolyte configuration (green squares in Figure 11A). 
Ion current measurements were also performed at a lower temperature when the conductivity cell was placed on ice. Lowering temperature decreased the electric mobility of ions so that the measured ion currents became lower. The normalized noise level at the two concentrations was however comparable (Figure 12).
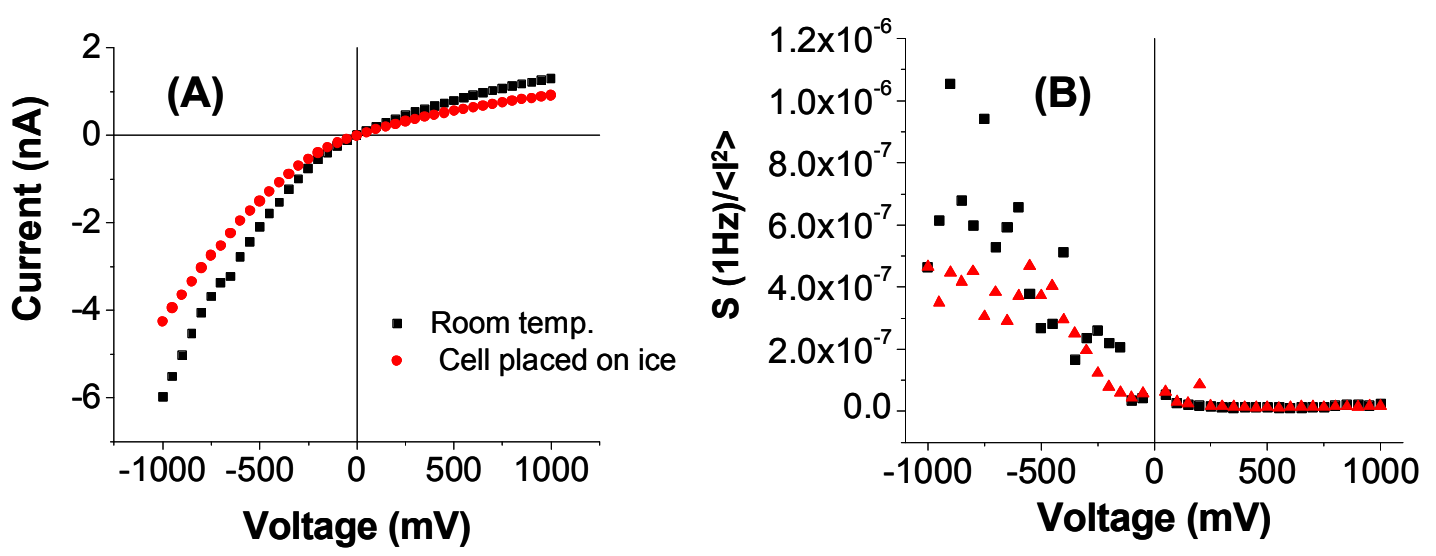

Figure 12. Ion current through a single conically shaped nanopore with a narrow opening of $38 \mathrm{~nm}$ in diameter studied at room temperature and at $0^{\circ} \mathrm{C}$. (A) Currentvoltage curve, $(B)$ Normalized power spectra of ion currents as a function of voltage.

The measurements of ion currents with solutions of a higher density and lower temperature suggested that the formation of vortices is an unlikely cause for the voltage-dependent properties of the $1 / f$ noise in polymer and glass nanopipettes.

\subsection{Modeling of electric field in conically shaped nanopores}

Although our experiments did not confirm the formation of vortices in conically shaped nanopores, we quantitatively described the electric fields to which ions and water molecules are subjected inside the pore. High electric fields might cause secondary effects, e.g. enhanced water dissociation ${ }^{41,42}$, which could lead to $\mathrm{pH}$ fluctuations in the pore, and consequently increase ion current fluctuations. ${ }^{43}$ According to the second-order Wiener effect, the dissociation of water is a strong function of the applied field, while the recombination was found to be electric field independent. Poisson-Nernst-Planck modeling of the electric fields was performed for the $7 \mathrm{~nm}$ in diameter nanopore for various concentrations of background electrolyte (Figure 13). 
Modeling revealed that the asymmetric shape of the conical nanopores in combination with the finite surface charge causes not only asymmetric ionic concentrations (Figure 5) but also results in a polarity dependent electric fields inside a nanopore. At lower concentrations of the background electrolyte, ions and water molecules experience very high electric fields up to $10 \mathrm{MV} / \mathrm{m}$ for negative voltages. For positive external voltages, the electric fields that the ions and water feel are much lower. The asymmetry of the electric field in conical nanopores and glass nanopipettes could indeed explain the voltage-dependent character of the $1 / f$ noise. Figure 14 shows the value of the electric field at the narrow opening of the pore as a function of the applied external voltage and background $\mathrm{KCl}$ concentration. Similar to the experimental data of the normalized noise, $\mathrm{S}(1 \mathrm{~Hz}) /<\mid>^{2}$ (Figures 2B-4B), the electric field increases steeply with the magnitude of the negative voltage. In addition, the electric field for positive voltages is lower for the lower $\mathrm{KCl}$ concentration. Our numerical simulations of the electric field at the pore narrow opening suggest that the normalized noise in conically shaped nanoporous structures might be directly related with the magnitude of the electric field. Symmetric SiN pores do not produce the electric field asymmetry and the $1 / f$ noise has an equilibrium character.
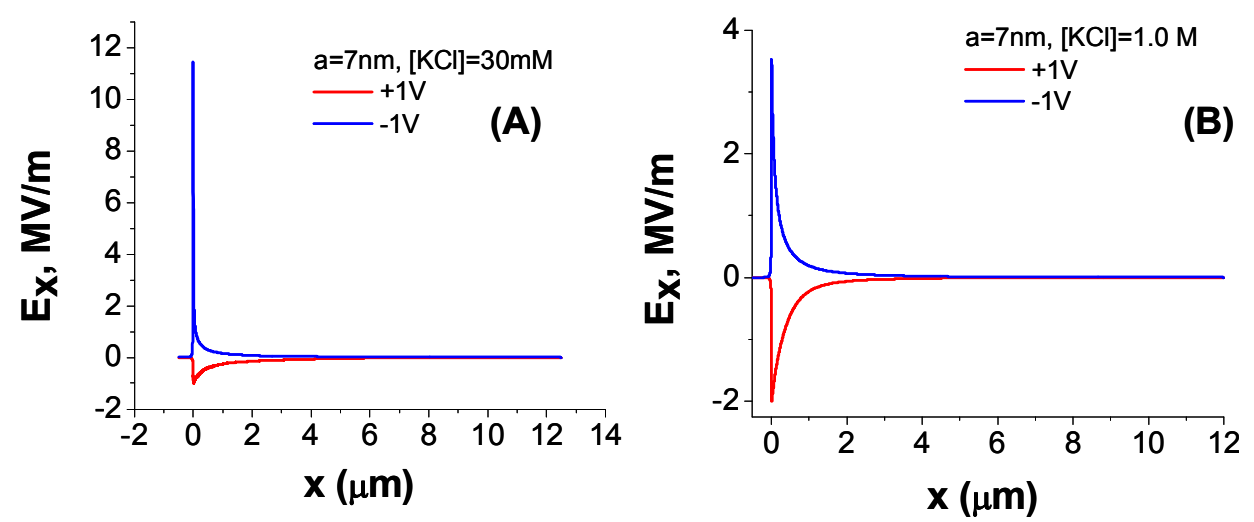

Figure 13. Numerical modeling of electric fields inside a conically shaped nanopore by the Poisson-Nernst-Planck equations. The electric field is dependent on the concentration of the background electrolyte. $(A)$ and $(B)$ present electric fields calculated for $30 \mathrm{mM}$ and $1.0 \mathrm{M} \mathrm{KCl}$ background electrolyte concentrations, respectively. Note also a strong position dependence of the field along the pore axis. The modeled nanopore was $12 \mu \mathrm{m}$ long, and had opening diameters of 7 and $500 \mathrm{~nm}$. 


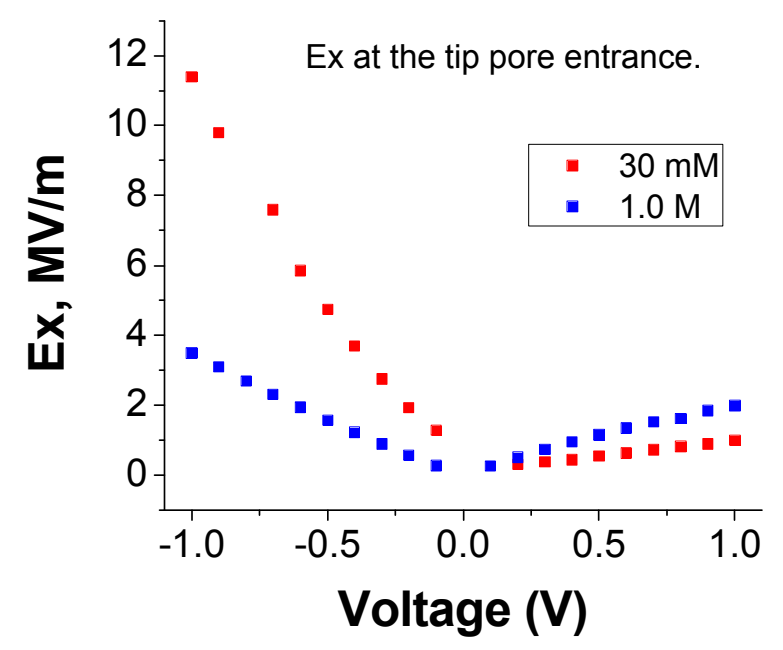

Figure 13. Magnitude of the electric field at the narrow opening of a conically shaped nanopore as a function of applied voltage at $30 \mathrm{mM}$ and $1 \mathrm{M} \mathrm{KCl}$. The graph was obtained by numerically solving the Poisson-Nernst-Planck equations for a conical nanopore, $12 \mu \mathrm{m}$ long, with openings of 7 and $500 \mathrm{~nm}$ in diameter.

\section{CONCLUSIONS}

We have studied ion currents through three rectifying nanopore structures: conically shaped nanopores in polymer films, glass nanopipettes, and cylindrical SiN nanopores coated with gold. We tested two hypotheses for the origin of non-equilibrium $1 / f$ noise in polymer nanopores. Namely, we analyzed whether the observed voltagedependence of the $1 / f$ noise magnitude could be explained by pore wall dynamics, and/or the formation of vortices and other patterns of fluid dynamics in the pore.

Ion currents through polymer rectifying pores recorded in solutions of various $\mathrm{KCl}$ concentrations, as well as ion currents through rigid rectifying SiN nanopores, indicate that pore wall dynamics might indeed impact the properties of ion current noise. But the system of glass nanopipettes, although rigid, was still characterized by the voltagedependent $1 / \mathrm{f}$ noise. In order to evaluate vortex formation in the studied voltage range, ion currents in polymer conical nanopores were recorded with elevated solution density, and decreased temperature. The voltage-dependence of the currents power spectra observed in these conditions suggests that vortices are unlikely to be formed in our system. The Poisson-Nernst-Planck modeling of electric fields in conically shaped 
nanopores however revealed the existence of high electric fields for one voltage polarity for which the normalized noise of ion currents showed a non-equilibrium character. The values of electric fields to which ions and water molecules are subjected were also found to be dependent on the background $\mathrm{KCl}$ concentration. We therefore postulate that the voltage-dependent $1 / \mathrm{f}$ noise could result from secondary effects originating from high electric fields, e.g. water dissociation.

It is also possible that $1 / \mathrm{f}$ noise in rectifying nanopore systems might not result from a single mechanism, but rather might originate from several effects, contributing in various systems to a different degree.

\section{Acknowledgements}

Single-ion irradiation at the Institute for Heavy lons Research, Darmstadt, Germany is greatly acknowledged. The research was supported by the National Science Foundation (CHE 0747237) and the UC System/National labs collaboration program. This work was performed under the auspices of the U.S. Department of Energy by Lawrence Livermore National Laboratory under Contract DE-AC52-07NA27344.

\section{REFERENCES}

1. Howorka, S.; Siwy, Z.S. Chem. Soc. Rev. 2009, 38, 2360.

2. Dekker, C. Nat Nanotechnol. 2007, 2, 209.

3. Healy, K. Nanomed. 2007, 2, 459.

4. Siwy, Z.; Howorka, S. Chem. Soc. Rev. 2010, 39, 1115.

5. Hanggi, P.; Marchesoni. F. Rev. Mod. Phys. 2009, 81, 387.

6. Hille, B. Ion Channels of Excitable Membranes, Sinauer Associates, 2001.

7. Siwy, Z.; Fulinski, A. Phys. Rev. Lett. 2002, 89, 158101.

8. Powell, M. R.; Sullivan, M.; Vlassiouk, I.; Constantin, D.; Sudre, O.; Martens, C. C.; Eisenberg, R. S.; Siwy, Z. S. Nat. Nanotechnol. 2008, 3, 51.

9. Kasianowicz, J. J.; Brandin, E.; Branton, D.; Deamer, D. W. Proc. Natl. Acad. Sci. U. S. A., 1996, 93, 13770.

10. Li, J.; Stein, D.; McMullan, C.; Branton, D.; Aziz, M.J.; Golovchenko, J.A. Nature 2001, 412, 166. 
11. Smeets, R. M.; Keyser, U. F.; Krapf, D.; Wu, M. Y.; Dekker, N. H.; Dekker, C. Nano Lett., 2006, 6, 89.

12. Kogan, Sh. Electronic Noise and Fluctuations in Solids, Cambridge University, London, 1996.

13. Bezrukov, S.M.; Winterhalter, M. Phys. Rev. Lett. 2000, 85, 202.

14. Smeets, R. M.; Keyser, U. F.; Dekker, N. H.; Dekker, C. Proc. Natl. Acad. Sci. USA 2008, 105, 417.

15. Smeets, R.M.M.; Dekker, N.H.; Dekker, C. Nanotechnology 2009, 20, 095501.

16. Hoogerheide, D.P.; Garaj, S.; Golovchenko, J.A. Phys. Rev. Lett. 2009, 102, 256804.

17. Chen, P.; Mitsui, T.; Farmer, D. B.; Golovchenko, J.; Gordon, R. G. Branton, D. Nano Lett. 2004, 4, 1333.

18. Powell, M.R.; Vlassiouk, I.; Martens, C.; Siwy, Z.S. Phys. Rev. Lett. 2009, 103, 248104

19. Powell, M.R.; Martens, C.; Siwy, Z.S. Chem. Phys. 2010, 375, 529.

20. Tasserit, C.; Koutsioubas, A.; Lairez, D.; Zalczer, G.; Clochard, M.-C. Phys. Rev. Lett. 2010, 105, 260602.

21. Nilsson, J.; Lee, J. R. I.; Ratto, T. V.; Letant, S. E. Adv. Mater. 2006, 18, 427.

22. Apel, P. Yu; Korchev, Y.E.; Siwy, Z.; Spohr, R.; Yoshida, M. Nucl. Instrum. and

Methods in Physics Research, Section B 2001, 184, 337.

23. Siwy, Z.; Fulinski, A. Phys. Rev. Lett. 2002, 89, 198103.

24. Wei, C.; Bard, A. J.; Feldberg, S. W. Anal. Chem. 1997, 69, 4627.

25. Umehara, S.; Pourmand, N.; Webb, C. D.; Davis, R. W.; Yasuda, K.; Karhanek, M.

Nano Lett. 2006, 6, 2486.

26. Sannomiya, T.; Dermutz, H.; Hafner, C.; Voros, J.; Dahlin, A. B. Langmuir 2010, 26, 7619.

27. Israelachvili, J.N. Intermolecular and Surface Forces, Academic, London, 1992.

28. Fleischer, R. L.; Price, P. B.; Walker, R. M. Nuclear Tracks in Solids. Principles and Applications, Univ. of California Press, Berkeley 1975.

29. Spohr, R. German Patent DE 2951376 C2, 1983; US Patent 4369370, 1983.

30. Vlassiouk, I.; Smirnov, S.; Siwy, Z.S. Nano Letters 2008, 8, 1978.

31. Vlassiouk, I.; Smirnov, S.; Siwy, Z.S. ACS Nano 2008, 2, 1589

32. Cervera, J.; Schiedt, B.; Ramirez, P. Europhys. Lett. 2005, 71, 35.

33. Cervera, J.; Schiedt, B.; Neumann, R.; Mafe, S.; Ramirez, P. J. Chem. Phys. 2006, 124, 104706.

34. Hooge, F.N. Phys. Lett. 1969, 29A, 139.

35. Vlassiouk, I.; Kozel, T.; Siwy, Z.S. J. Am. Chem. Soc. 2009, 131, 8211-8220.

36. Ramirez, P.; Apel, P.Y.; Cervera, J.; Mafe, S. Nanotechnology 2008, $19,315707$.

37. Rubinstein, I.; Shtilman, L. J. Chem. Soc. Faraday Trans. 1979, 2, 75.

38. Yossifon, G.; Chang, H-C. Phys. Rev. Lett. 2008, 101, 254501.

39. Daiguji, H.; Oka, Y.; Shirono, K. Nano Lett. 2005, 5, 2274.

40. Fologea, D.; Uplinger, J.; Thomas, B.; McNabb, D.S.; Li, K. Nano Lett. 2005, 5, 1734.

41. Bassignana, I.C.; Reiss, H. J. Membr. Sci. 1983, 15, 27.

42. Mafe, S.; Ramirez, P. Acta. Polym. 1997, 48, 234-250. 
43. Korchev, Y. E.; Bashford, C. L.; Alder, G. M.; Apel, P. Y.; Edmonds, D. T.; Lev, A. A.; Nandi, K.; Zima, A. V.; Pasternak, C. A. FASEB J. 1997, 11, 600. 